\title{
Fusion strategies for selecting multiple tuning parameters for multivariate calibration and other penalty based processes: A model updating application for pharmaceutical analysis
}

\author{
Alister J. Tencate ${ }^{\mathrm{a}}$, John H. Kalivas ${ }^{\mathrm{a} *}$, Alexander J. White ${ }^{\mathrm{b}}$ \\ ${ }^{a}$ Department of Chemistry, Idaho State University, Pocatello, Idaho 83209, USA \\ ${ }^{\mathrm{b}}$ Department of Physics and Optical Engineering, Rose-Hulman Institute of Technology, Terre Huate, IN 47803, \\ USA \\ *Corresponding author. Tel.: +1 208-282-2726; fax +1 208-282-4373. E-mail address: kalijohn@isu.edu (J.H. \\ Kalivas)
}

\begin{abstract}
New multivariate calibration methods and other processes are being developed that require selection of multiple tuning parameter (penalty) values to form the final model. With one or more tuning parameters, using only one measure of model quality to select final tuning parameter values is not sufficient. Optimization of several model quality measures is challenging. Thus, three fusion ranking methods are investigated for simultaneous assessment of multiple measures of model quality for selecting tuning parameter values. One is a supervised learning fusion rule named sum of ranking differences (SRD). The other two are non-supervised learning processes based on the sum and median operations. The effect of the number of models evaluated on the three fusion rules are also evaluated using three procedures. One procedure uses all models from all possible combinations of the tuning parameters. To reduce the number of
\end{abstract}


models evaluated, an iterative process (only applicable to SRD) is applied and thresholding a model quality measure before applying the fusion rules is also used. A near infrared pharmaceutical data set requiring model updating is used to evaluate the three fusion rules. In this case, calibration of the primary conditions is for the active pharmaceutical ingredient (API) of tablets produced in a laboratory. The secondary conditions for calibration updating is for tablets produced in the full batch setting. Two model updating processes requiring selection of two unique tuning parameter values are studied. One is based on Tikhonov regularization (TR) and the other is a variation of partial least squares (PLS). The three fusion methods are shown to provide equivalent and acceptable results allowing automatic selection of the tuning parameter values. Best tuning parameter values are selected when model quality measures used with the fusion rules are for the small secondary sample set used to form the updated models. In this model updating situation, evaluation of all possible models, thresholding, and iterative SRD performed equivalently for the three fusion rules with TR and PLS performed worse. While the application is model updating, the fusion processes are applicable to other situations requiring selection of multiple tuning parameter values.

\section{Keywords:}

Sum of ranking differences; Multivariate calibration; Partial least squares; Tikhonov regularization; Calibration maintenance; Model updating; Fusion; Multicriteria optimization; Penalty regression 


\section{Introduction}

A growing trend in developing new multivariate calibration methods and other data analysis modeling processes is including additional penalty terms in the model. Each additional penalty term typically involves a tuning parameter requiring optimization for an acceptable model. Since the goals of many calibration models are accurate and precise predications for new samples, it seems reasonable to use a model quality measure based on prediction error, such as from a cross-validation process. However, assessing the model quality with one measure based on prediction error when multiple tuning parameters are involved increases the risk of overfitting. To counter the chance of overfitting, additional model quality measures should be evaluated as tuning parameters are adjusted. With the increase of model quality measures comes the need for decision making tools to evaluate the multiple model quality measure allowing automatic selection of multiple tuning parameters. This situation is becoming increasingly relevant for a wide range of disciplines.

One approach to optimizing a multipenalty based model using multiple measures of model quality is multicriteria (multiresponse) optimization [1-7]. Broadly speaking, multicriteria optimization involves "Making a systematic and rational decision of the best alternative among several candidates when multiple (and often conflicting) criteria are present.” [1]. The difficult part is how to combine (evaluate) and possibly weight the multiple criteria (measures of model quality in this paper) to form the final selection. Several approaches have been used to try and solve this issue. Popular are desirability functions [4]. However, there are several functions to choose from as well as numerous weighting schemes. An approach to avoid evaluation functions and weighting schemes is target optimization. However, like some desirability functions, target values for the model quality measures are needed and often this is not possible. Some graphical 
empirical approaches have been suggested, but these require expert knowledge and experience with the graphical interpretation [8-13].

Data fusion is becoming more common place across multiple disciplines in order to provide improved prediction accuracy for quantitative (e.g., concentrations) and qualitative (e.g., classification) purposes [14]. In the broadest meaning, "data fusion is a multilevel, multifaceted process dealing with the automatic detection, association, correlation, estimation, and combination of data and information from multiple sources." [15]. Three levels of fusion are generally considered: low, mid, and high. A recent review paper characterizes these categories and provides a thorough review of fusion methods [16]. Briefly, low level fusion involves concatenation of data arrays to a single array. Mid-level indicates that features are extracted from each data array and then the data arrays are concatenated to a single array. The third level is termed high where multiple modeling processes are used for one or more data arrays and the final prediction result is a combination of individual results from using each model individually. The approach taken in this paper for tuning parameter selection probably fits high level fusion best. In this case, the final result (selected tuning parameter values and hence, model) is based on a fusion of the multiple inputs (model quality measures) where each input could be used separately to make the final decision. No weighting scheme is used for the fusion processes. The method of sum of ranking differences (SRD) $[17,18]$ has recently been used as a process allowing automatic tuning parameter selection for the calibration methods partial least squares (PLS) and the Tikhonov regularization (TR) method known as ridge regression (RR) [19]. The SRD method uses a collection of model quality measures to form a consensus model ranking by comparing each model quality measure value to an assigned target direction, such as minimum, maximum, etc., but a specific numerical value can also be used. The smaller the 
assigned rank given a model, the closer the corresponding model meets the respective targets of the different model quality measures. A single model or a collection of models can then be selected based on the SRD ranks.

While SRD prefers at least seven model quality measures for meaningful rankings, fusion rules such as mean, sum, stacking, and others [20-23] are not constrained in this way. However, these fusion rules lack the directional targeting used with SRD in the ranking process. The SRD process also includes a comparison to random ranking [18] in order to ascertain if a model ranking is any different than randomly ranking that model. For this study, the three fusion rules SRD, sum, and median are evaluated.

To test the three fusion rules and fusion processes developed in this paper for multiple tuning parameter selection, a model updating situation is studied. Common in model updating, a large calibration sample set characterizing the primary conditions is used to build a calibration model for the primary conditions. A small updating set of samples from new secondary conditions are then used to update the primary calibration model to focus on predicting new samples from the secondary conditions. This approach to model updating usually necessitates optimization of at least two tuning parameters [9-11,24].

Two model updating methods are studied and both require selection of two tuning parameter values. One is a Tikhonov regularization (TR) method [9-11]. The other method is based on partial least squares (PLS) [24]. A near infrared (NIR) pharmaceutical data set requiring model updating is used to evaluate the three fusion rules for selecting respective model tuning parameter values. In this data set, the primary conditions are for calibration of the active pharmaceutical ingredient (API) of tablets produced in a laboratory and the secondary conditions 
requiring model updating are tablets to be predicted for API content produced in a full batch setting.

\section{Methods}

\subsection{Model Updating}

\subsubsection{TR}

The TR model updating process involves a variation on the standard form of TR, ridge regression (RR) [9-11]. For TR model updating, the RR model for the primary conditions is updated by augmenting the primary $\mathbf{X}, \mathbf{y}$ set with the secondary set $\mathbf{M}, \mathbf{y}_{\mathbf{M}}$ composed of a few samples measured in the secondary conditions as shown in equation (1).

$$
\left(\begin{array}{c}
\mathbf{y} \\
\mathbf{0} \\
\eta \mathbf{y}_{\mathbf{M}}
\end{array}\right)=\left(\begin{array}{c}
\mathbf{X} \\
\lambda \mathbf{I} \\
\eta \mathbf{M}
\end{array}\right) \mathbf{b}
$$

where $\mathbf{X}$ represents the $m \times n$ primary calibration matrix for $m$ samples measured at $n$ variables (wavelengths for spectral data), $\mathbf{y}$ denotes the $m \times 1$ vector with the corresponding calibration reference values, $\mathbf{M}$ symbolizes the $s \times n$ matrix composed of $s$ updating samples from the secondary conditions with $\mathbf{y}_{\mathbf{M}}$ reference values $(s \times 1)$, $\mathbf{I}$ is the usual notation for the identity matrix of size $n \times n, \mathbf{0}$ signifies the $n \times 1$ zero vector, and $\mathbf{b}$ designates the model regression vector to be estimated as adjusted by tuning parameter values for $\lambda$ and $\eta$ in the range $0 \leq \lambda, \eta<\infty$. The solution to equation (1)

$$
\hat{\mathbf{b}}=\left(\mathbf{X}^{T} \mathbf{X}+\lambda^{2} \mathbf{I}+\eta^{2} \mathbf{M}^{T} \mathbf{M}\right)^{-1}\left(\mathbf{X}^{T} \mathbf{y}+\eta^{2} \mathbf{M y}_{\mathbf{m}}\right)
$$

results from the minimization of expression

$$
\min \left(\|\mathbf{X b}-\mathbf{y}\|^{2}+\lambda^{2}\|\mathbf{b}\|^{2}+\eta^{2}\left\|\mathbf{M b}-\mathbf{y}_{\mathbf{m}}\right\|^{2}\right)
$$


where the hat on $\mathbf{b}$ indicates an estimated value and $\|\cdot\|$ denotes the $L_{2}$ vector norm (Euclidean norm). As illustrated in the minimization condition shown in expression (3), the model vector is constrained to accurately predict under both primary and secondary conditions. However, depending on the tuning parameter values, the model vector can be biased towards the primary or secondary conditions by the respective tuning parameter values. Specifically, the primary calibration set is designed such that there are enough samples to build an acceptable primary calibration model, but there are only a few samples from the secondary conditions (not enough to build a calibration model). The $\eta$ tuning parameter weights the secondary samples in order to not be outweighed by the large number of primary samples.

\subsubsection{PLS}

The PLS approach to model updating parallels the TR approach detailed above, but the PLS latent variables (LVs) replace the $\lambda$ tuning parameter forming the model

$$
\left(\begin{array}{c}
\mathbf{y} \\
\eta \mathbf{y}_{\mathbf{M}}
\end{array}\right)=\left(\begin{array}{c}
\mathbf{X} \\
\eta \mathbf{M}
\end{array}\right) \mathbf{b}
$$

This form of PLS is similar to recursive PLS [24] except in equation (4), the tuning parameter is on the small set of secondary samples instead of on the lager primary condition set of samples. In order to update a calibration model, this PLS approach, much like the TR method, generates a single model vector constrained to predict well under both primary and secondary conditions. There are tradeoffs to using PLS for model updating compared to TR. The PLS model updating process is generally faster due the LVs being much more limited than the number of possible $\lambda$ tuning parameter values, albeit, with TR, the user has the flexibility to set the range and number 
of $\lambda$ values. However, the discrete nature of the LVs can potentially hamper identifying the better models due to the limited possibilities as observed in previous work [25].

\subsection{Model quality measures}

A number of bias and variance model quality measures are evaluated with the fusion rules in order to determine a good updated calibration model (tuning parameter values). For this study, prediction bias refers to model prediction accuracy and prediction variance corresponds to prediction precision (uncertainty). The goal of the model quality measures is to assess the degree of overfitting (lower bias but greater variance or model complexity) versus underfitting (greater bias but less variance or model complexity). Thus, the task of the fusion rules is to identify those models with an acceptable bias/variance tradeoff [26-28].

It is possible to combine bias and variance measures into one quality measure to generate U shaped curves where the better tuning parameters are at the minimum of the U-curve allowing automatic model selection [26-28]. This is in contrast to plotting bias measures against variance measures to form L shaped curves requiring assessment of the corner region of the L-curve for the better tuning parameters.

This investigation utilizes bias merits, variance (or model complexity) quality measures, and U-curve quality measures. A bias measure used is the prediction error (accuracy) for a collection of samples labeled as the root-mean-square-error

$$
\mathrm{RMSE}=\sqrt{\sum_{i=1}^{p}\left(y_{i}-\hat{y}_{i}\right)^{2} / p}
$$

relative to the specific sample set of $p$ samples. For this study, equation (5) will be used for the primary calibration samples (RMSEC) using $\mathbf{X}$ and $\mathbf{y}$ with $p=m$, the secondary updating set (RMSEM) using $\mathbf{M}$ and $\mathbf{y}_{\mathbf{M}}$ with $p=s$, and a separate set of secondary samples used for the 
secondary validation samples (RMSEV). The secondary validation samples are not used to form or select the updated model. The corresponding measures $\mathrm{R}^{2}$, slope, and y-intercept from plotting predicted values against reference values are also evaluated. A key measure used to characterize model complexity and hence the potential variance (prediction uncertainty) is the model vector $\mathrm{L}_{2}$ norm symbolized by $\|\hat{\mathbf{b}}\|[26-31]$. The jaggedness (J) of a spectroscopic model vector is also related to complexity and the degree of overfitting [27] and is evaluated as a model quality measures. It is computed for the $i$ th tuning parameter (model) by

$$
\mathbf{J}_{i}=\sqrt{\sum_{j=2}^{n}\left(\mathbf{b}_{i j}-\mathbf{b}_{i(j-1)}\right)^{2}}
$$

over the $n$ wavelengths. The $\mathrm{U}$-curves are formed by

$$
\mathrm{C}_{i}=\left(\frac{\|\mathbf{b}\|_{i}-\|\mathbf{b}\|_{\text {min }}}{\|\mathbf{b}\|_{\text {max }}-\|\mathbf{b}\|_{\text {min }}}\right)+\left(\frac{\mathrm{RMSEC}_{i}-\mathrm{RMSEC}_{\text {min }}}{\mathrm{RMSEC}_{\text {max }}-\mathrm{RMSEC}_{\text {min }}}\right)
$$

where respective values in the parenthesis are scaled to range between 0 and 1 inclusive. Three other variations of equation (7) were evaluated where RMSEM replaces RMSEC and the corresponding combinations with $\mathrm{J}$ replacing the $\mathrm{L}_{2}$ norm.

\subsubsection{Final combinations of model quality measure for the fusion rules}

A previous study on selecting a single tuning parameter, e.g., LVs for PLS, showed that models ranked lowest by SRD can be influenced by the choice of quality measures included in the SRD process [19]. The variation is small and reduces as more measures are used. This model variation is similar to different models being selected by different single model criteria and/or different cross-validation methods. The final combination of model quality measures is up to the user. For the fusion rules, there should be a balance between the bias and variance measures so 
as not to weight the model selection toward under or overfitted models. Thus, numerous

combinations of model measures for the primary and secondary conditions were evaluated. As noted in section 4.1, it was found that combinations of model quality measures only characterizing the secondary conditions consistently identified models predicting best for the secondary validation samples. Including measures for the primary conditions, such as RMSEC, tended to drive the fusion rules to rank models as best with too much compromise between the primary and secondary conditions. A model compromising the primary and secondary conditions does not predict new samples for the secondary condition as well as a model tuned to predicting samples from the secondary conditions. Thus, the final measures used for the fusion rules were: $\mathrm{R}^{2}$ and slope from plotting the secondary updating sample predicted values $\left(\hat{\mathbf{y}}_{\mathbf{M}}\right)$ against reference values ( $\left.\mathbf{y}_{\mathbf{M}}\right)$, RMSEM, model vector $\mathrm{L}_{2}$ norm, $\mathrm{J}$, and $\mathrm{C}$ from equation (7) replacing RMSEC with RMSEM and then the same but with $\mathrm{J}$ instead of the $\mathrm{L}_{2}$ norm for a total of seven measures. For the SRD process, target values for all measures were set to minimization. Model quality measures, such as $\mathrm{R}^{2}$, normally maximized are reconfigured to minimization. In the case of $\mathrm{R}^{2}$, values are subtracted from 1 . See the next section for further explanation setting SRD target values.

\subsection{Fusion strategies for model ranking}

In order to apply the fusion rules, a data matrix with $q$ rows of model quality measures and $t$ columns of model tuning parameters (or combinations of tuning parameters for two or more model tuning parameters) is formed. This matrix is then subjected to the fusion rules to rank the columns (models) relative to the model quality measures used for the matrix rows. For this study, each column is either a $\lambda, \eta$ or LV, $\eta$ combination for TR or PLS respectively, albeit, 
TR and PLS can be combined in the matrix for simultaneous model selection and comparison [19].

Because model quality measures are generally not measured on the same scale, the values can be scaled to have similar magnitudes. Numerous scaling approaches are possible such as range scaling inclusively between 0 and 1 , autoscaling (or standardization) to mean 0 and standard deviation 1, pre-transforming to row-wise numerical rank values, and others [32]. Experience with SRD for model selection indicates that row normalization works best [19]. Thus, in this study, values in each model measure row were normalized to unit length and for the sum and median fusion rules, values were row-wise pre-ranked.

As previously noted, SRD has been tested as a fusion rule to rank and select tuning parameter values for TR and PLS [19] and as such, it is the primary ranking method studied. The SRD process is well described [17,18] but a brief overview is provided and depicted in Figure 1. In the context of this study and with reference to the graphic in Figure 1, target directions are required for the each model measure row, e.g., minimum, maximum, median, or mean of respective rows or known reference values can be used. Next, for each row of this model quality matrix to be evaluated by the SRD fusion rule, the column (model) value closest to the corresponding row target is identified. A target vector is then created with these values sorted (ranked) from low to high and respective row indexes are noted. The matrix of model quality measures is then rearranged to this target row index sort and all values in each respective column (model) are ranked from low to high. The absolute value of the difference between the target row ranking and each column ranking of the reordered rows is computed and summed for each column to form the column-wise vector of the final SRD ranked models. Recently, SRD has modified to handle observations with ties [33] and has been related to the inversion number [34]. 
The data fusion rules sum and median [21] are also evaluated. With the sum rule, model quality measure values for each column (model) are summed to form the final model ranking. In the median process, the median in each column is used to rank the corresponding model. The model with the lowest rank is deemed best. Presented in Figure 2 is a graphical illustration of a generic fusion rule to the model quality matrix.

\subsection{Number of models evaluated by the fusion rules}

As the number of tuning parameters increases with development of new penalty regression and modeling methods, evaluating all possible models at once may hinder the fusion processes. For example, the TR and PLS model updating processes require two parameters each. Thus, each model quality measure can be viewed as a landscape of sizes $\lambda$ by $\eta$ for TR and LVs by $\eta$ for PLS. For analysis by the fusion rules, each model quality measure is unfolded to a row vector of length $\lambda(\mathrm{LVs})$ times $\eta$ for the corresponding combinations of $\lambda(\mathrm{LVs})$ and $\eta$. The model quality row vectors are assembled into a matrix for the fusion rules to rank the columns (models). Thus, before applying the fusion rules, it may be advantageous to remove poorly predicting models from consideration. This is similar to the spectral calibration situation where non-informative wavelengths can be removed before forming the final calibration model. Two approaches are evaluated to pre-filter the models and are described next.

\subsubsection{Using all possible models}

As a basis for comparison, the fusion rules were used on the complete set of possible models formed using the full range of tuning parameter values. This approach has been shown to 
be reliable when selecting single model tuning parameters for methods such as RR and PLS and other processes [19]. This approach is evaluated in this study as well.

\subsubsection{Thresholding models}

A simple procedure to reduce the number of models for the fusion rules to evaluate is to incorporate thresholds on model quality measures to identify the better models [25] and then move forward to the fusion rules. Rather than fine tuning thresholds for several model measures, in this study, two coarse thresholds are used on one model measure to exclude models with extremely poor predictions as well as models that are clearly overfitted to the calibration samples.

The final single measure found to work best for the coarse thresholding is the $\mathrm{R}^{2}$ for the update samples from the secondary conditions $\left(\mathbf{M}, \mathbf{y}_{\mathbf{M}}\right)$. Thresholds can be set different ways. For example, a threshold can be set to a particular value of $\mathrm{R}^{2}$ or it can be set to remove a specified percent of the models generated. The approach in this paper is to remove percentages of models. Values evaluated were removal of $50 \%$ and $70 \%$ of the models with the lowest update set $\mathrm{R}^{2}$. Also studied was setting an upper percent threshold to remove $10 \%$ of the models with the highest update set $\mathrm{R}^{2}$ (in order to eliminate overfitted models). Thus, four different combinations of thresholds were investigated. A representation of the thresholding process is shown in Figure 3. The models remaining are then passed on to the fusion rules using multiple quality measures. 


\subsubsection{Iterative $S R D$}

One of the features of the SRD fusion process is a distribution of random rankings providing the probability of obtaining randomly ranked models $[4,5,19]$. If SRD ranks are not in the distribution, then these ranks are considered statistically significant. The position of a rank relative to the distribution can be used as a criterion for retaining models iteratively. Specifically, a subset of models are collected based on ranks from the initial SRD fusion rule and the SRD process is repeated on these retained models. In this study, the iterations continue until no more than 100 models remain. The remaining models are then ranked a final time. Models kept from the initial and each iteration of SRD must have ranks at least two standard deviations smaller than the mean rank of the random ranking distribution.

\subsection{Validating model rankings}

A cross-validation $(\mathrm{CV})$ process is performed on the matrix of model quality values to validate the fusion ranking results $[18,19]$. The goal is to assess the consistency of the fusion rankings when some model quality measures are removed before fusion is applied. Depending on the number of model measures used for ranking, different CV schemes can be implemented. Due to the limited number of model quality measures used (only seven as per section 2.2.1), leaveone-out CV is used with the fusion methods. Specifically, one model quality measure (row) is removed from the model quality matrix. The fusion rules are applied to rank the models. The removed row is replaced and the next row is removed and the models are ranked again. This process is repeated until all model quality measure has been left out once. From the CV, obtainable are uncertainties in the model rankings, boxplots of the fusion rankings, as well as, if desired, statistical testing of the rankings $[18,19]$. In this study, statistical testing of the rankings 
was not performed. Due to the complexity of the situation and number of models, the boxplots are not shown. In order to further enhance the rankings, the CV rank reliability measure (RRM) is computed for the ith model as

$$
\mathrm{RRM}_{i}=\left(\frac{\sigma_{\mathrm{FR}_{i}}-\sigma_{\mathrm{FR}_{\text {min }}}}{\sigma_{\mathrm{FR}_{\text {max }}}-\sigma_{\mathrm{FR}_{\text {min }}}}\right)+\left(\frac{\overline{\mathrm{FR}}_{i}-\overline{\mathrm{FR}}_{\text {min }}}{\overline{\mathrm{FR}}_{\text {max }}-\overline{\mathrm{FR}}_{\text {min }}}\right)
$$

where the fusion rule (FR) ranking standard deviation $\left(\sigma_{\mathrm{FR}_{i}}\right)$ and mean fusion rule $\left(\overline{\mathrm{FR}}_{i}\right)$ values are ranged scaled to vary between 0 and 1 inclusive using the minimum and maximum values for the corresponding $i$ th model. The RRM provides a rank for each model that factors in the mean and standard deviations of the ranks from the $\mathrm{CV}$, both of which are equally weighted. By selecting the model that is ranked low by RRM, the final model will ideally be one that has a low and consistent rank. The RRM is used for ranking all models in this study.

\section{Experimental}

\subsection{Algorithms}

All algorithms for TR and PLS for model updating, fusion, and model quality measures were written by the authors using MATLAB 8.1 (The Math Works, Natick, MA). The MATLAB SRD algorithm is available for downloading at [35].

\subsection{NIR pharmaceutical data and models}

Spectra were measured for Escitalopram tablets at 404 wavelengths ranging from approximately 950 to $1350 \mathrm{~nm}$. The tablets are categorized based on weight yielding four different tablet types. Two of the types are numbered types 1 and 2 for 90 and $125 \mathrm{mg}$, respectively. Available are spectra and reference values for 30 samples of each tablet type from 
three production batches [36,37]. The active pharmaceutical ingredient (API) content for each tablet is provided in units of percent weight. This investigation focused on using the same experimental design previously used [11]; specifically, updating a primary model for laboratory batch tablet types 1 and 2 to predict tablet types 1 and 2 measured in the secondary full production batch conditions. Briefly, a random 30 samples (15 tablets of type 1 and 2 each from the primary conditions) form the primary calibration set $(\mathbf{X}, \mathbf{y})$ and a random 4 samples (2 tablets type 1 and 2 each from the secondary conditions) are used for the secondary update set (M, $\mathbf{M} \mathbf{M})$. Of the remaining samples in the full production batch (secondary conditions), a random 30 (15 each from tablet types 1 and 2) are used for the validation set. This study only investigated a single random split to select the calibration and the update samples as in the previous work [11]. Samples in the primary calibration set and secondary updating set are locally mean centered to respective conditions. The validation samples are mean centered to the updating set.

The $\eta$ and $\lambda$ TR tuning parameter values range exponentially decreasing from 1,000 to 0 for 100 and 80 values respectively. The PLS algorithm uses the same $\eta$ values as listed for TR. For TR, the $\lambda$ and $\eta$ values resulted in 8,000 models and for PLS with 33 LVs, 3,300 models are formed.

\section{Results and discussion}

Discussed first are results using TR with and without pre-filtering the number of models for ranking by the fusion rules. Following this discussion, results using PLS are briefly presented as the results are similar to the TR results except for the effect from the discreet nature of PLS LVs. 


\subsection{General TR evaluation}

Shown in Figure 4 are some model quality measures characterizing primary and secondary TR prediction errors and the model $\mathrm{L}_{2}$ norms [11]. From these measures, it appears that the respective RMSE minima for the update set and secondary validation samples are in different model locations. Specifically, the RMSEM values continue to reduce as the weight on the secondary updating set increases tending towards models overfitted to the secondary updating set. Conversely, the RMSEV values for the secondary validation samples in the same region have started to increase indicating the models are indeed overfitted to the secondary updating sample. The guard against overfitting to the secondary updating sets is that the largest values for the $\mathrm{L}_{2}$ model norms are in the overfitted region. Thus, including this model quality measure is key to selecting tuning parameters balancing the bias/variance tradeoff. The RMSEC values for the primary calibration samples show different trends from the RMSEM and RMSEV. Thus, it is expected that the combinations of model quality measures favoring the update samples will generally select models better at predicting the secondary validation samples.

While many model quality measure combinations were studied for selecting TR models, it was found that using measures characterizing the secondary updating conditions does lead to selecting models with the low RMSEV values. For example, when using measures representing the primary calibration samples and the secondary samples, the fusion rules would rank models balancing prediction errors between primary calibration samples and secondary updating samples, the exact intent of expression (3). Specifically, the goal of expression (3) is to form models that predict samples from both the primary and secondary conditions. With proper tuning parameter values, models can be formed satisfying expression (3) that will be biased towards predicting the secondary samples at a sacrifice to predicting samples from the primary 
conditions. While empirical approaches to identifying these tuning parameters and models have been useful in past [9-11,25], using the fusion rules accomplishes exactly what is requested when model quality measures are used for both conditions. Specifically, the fusion rules rank models based on how well each model meets the model measures being evaluated for both conditions. However, the corresponding RMSEV values for these low ranked balanced models were not acceptable. If measures representing both the primary and secondary conditions are included, then a compromise model should indeed be expected and as noted, was obtained. A weighting scheme could be used for the measures to distinguish the secondary conditions. Rather than trying to discern a weighting scheme that could be data set dependent from the multitude of weighting possibilities, only measures for the secondary updating set are used to guide the fusion rules to select models better predicting the secondary validation samples. Thus, as noted in section 2.2.1., results tabulated in this paper are based on only using the fusion rules with the measures: $\mathrm{R}^{2}$ and slope from plotting the secondary updating sample predicted values against reference values, RMSEM, model vector $\mathrm{L}_{2}$ norm, J, and $\mathrm{C}$ from equation (7) but with RMSEM replacing RMSEC and then the same $\mathrm{C}$ but with $\mathrm{J}$ instead of the $\mathrm{L}_{2}$ norm. Using the $\mathrm{L}_{2}$ norm, $\mathrm{J}$, and $\mathrm{C}$ protects the fusion rules from ranking overfitted models as best.

\subsection{All possible TR models}

Presented in Table 1 are the results from the final lowest ranked models obtained by the three fusion rules on all 8,000 TR models. The lowest five ranked models by each fusion rule are plotted on the RMSEV landscape in Figure 5a. The tabulated results show that the lowest ranked models are acceptable. While the absolute best models are not identified, models immediately surrounding the ideal zone obtain the lowest rank values. As noted in past TR work [9-11,25] 
and evident in Figures 5 and 6, multiple models with nearly the same performance can be formed. That is, a neutral parameter set [38] can exist where several tuning parameter combinations form models with more or less, the same outputs.

\subsection{Thresholding TR models}

A number of thresholds can be used to reduce the number models evaluated by the fusion rules, e.g., retain a subset of all the models by retaining the top $\mathrm{X} \%$ models relative to some model quality measure. Because only the best model subset would be retained in this case, a second threshold would be needed to remove the top models thereby reducing the number of overfitted models. A variation of thresholds were studied and values presented in Table 1 are based on keeping the top $50 \%$ models minus the top $10 \%$ leaving approximately 3,200 models. This subset was based on only the $\mathrm{R}^{2}$ values for the small updating set of samples. This type of thresholding can be considered a gross filtering mechanism to remove the obvious non-ideal models from the selection process. From investigating variations in percentages for the thresholds, the SRD fusion rule was found to be more sensitive to the percentages used.

Results listed in Table 1 are from the lowest ranked models obtained by the three fusion rules on the 3,200 models and show that essentially the same results are obtained from when ranking all 8,000 models, albeit, different models are selected. The respective lowest five ranked models for each fusion rule are noted on the RMSEV landscape pictured in Figure 5b. Again, the absolute best models are not identified, but models immediately surrounding the best models are ranked lowest. 


\subsection{Iterative $S R D$}

Shown in Table 1 are the results from using the iterative SRD approach as previously described. Again, the results are essentially the same as before. After two SRD evaluations, the iterations were stopped as there were only four models left (100 was set as the maximum for stopping rule). The RMSEV image in Figure 6 shows the 332 models carried forward that were two standard deviations smaller than the mean rank of the random ranking distribution from the first SRD ranking.

\subsection{PLS}

The model quality measures for the models generated by the PLS algorithm show similar trends to those generated by TR (compare Figures 4 and 7). Analogous to TR, focusing on model quality measures based on only the updating samples from the secondary conditions with the fusion rules was best. However, as expected, the PLS model quality measures appear much more discrete across the LVs than the TR model measures do across the values for the ridge parameter $\lambda$. This discreteness can sometimes be problematic depending on the data set and situation [25]. For the situation shown in Figure 7, the region with low RMSEV values for PLS is much smaller than the TR counterpart. Thus, the number of possible models to identify that maintain satisfactory RMSEV values is reduced and ranking the models from this smaller zone as best becomes more difficult. For this reason, none of the models selected with the fusion rules have RMSEV values below 0.3300 (see Table 2). The five lowest ranked models for the three fusion rules based on all possible PLS models are identified on the RMSEV image in Figure 5c. It should be noted that the lowest possible PLS RMSEV values are similar to the lowest TR RMSEV values at 0.2300 . 


\section{Conclusion}

The tuning parameter selection procedures evaluated have been shown to successfully choose models with relatively low prediction errors for the validation samples from the secondary conditions. The processes are general and should be applicable to methods with more than two tuning parameters. Results presented are similar to those previously obtained $[11,25]$ using the same data split. Because the processes evaluated in this study can be automated, they can be applied to each split from using cross-validation, e.g., cross-validation of the secondary updating and validation samples. However, in the real world with model updating, one does not have the luxury to use cross-validation. As with most multicriteria optimization and some fusion methods, a weighting scheme of each model quality measure could be included before fusion. Similarly, a weighted fusion rule could be used. To keep the processes simple in this paper, no weighting methods were evaluated.

It should be noted that when using only one bias measure (RMSEM) and one variance measure (the model vector $\mathrm{L}_{2}$ norm) suboptimal models were selected. As noted in previous work [19], using a limited number of measures generally ranks model less accurately than using more.

It should also be noted that other approaches than the two studied in this paper can be used to reduce the number of models prior to application of the fusion rules. For example, the model quality measure landscapes could be divided into sections based on tuning parameter ranges. The models in each section would then be independently ranked using fusion rules. Collections of the lowest ranked models from in each section would be formed and then simultaneously re-ranked. This procedure is designed to isolate the best regions from each respective section of the model quality measure landscapes and then compare (re-rank) just these 
models. Some sectioning was applied to the data situation in this paper and no noticeable difference was observed between the final selected models and those presented previously.

\section{Acknowledgements}

This material is based upon work supported by the National Science Foundation under Grant No. CHE-1111053 (co-funded by MPS Chemistry and the OCI Venture Fund) and is gratefully acknowledged by the authors. This research was also partially supported by NSF Award 1156879 through the Department of Physics Research Experience for Undergraduates summer program. 


\section{References}

[1] M. Pavan, R. Todeschini, Scientific Data Ranking Methods: Theory and Applications, vol. 27, Elsevier, 2008.

[2] Grisoni, V. Consonni, S. Nembri, R. Todeschini, How to weight Hasse matrices and reduce incomparabilities, Chemom. Intell. Lab. Sys. 147 (2015) 95-104.

[3] N. Costa, J. Lourenço, A comparative study of multiresponse optimization criteria working ability, Chemom. Intell. Lab. Sys. 138 (2014) 171-177.

[4] N.R. Costa, J. Lourenço, Z.L. Pereira, Desirability function approach: A review and performance evaluation in adverse conditions, Chemom. Intell. Lab. Sys. 107 (2011) 234-244.

[5] N.R. Costa, Z.L. Pereira, Multiple response optimization; A global criterion-based method, J. Chemom. 24 (2010) 333-342.

[6] M.M.W.B. Hendriks, J.H. de Boer, A. Smilde, D.A. Doornbos, Multicriteria decision making, Chemom. Intell. Lab. Sys. 16 (1992) 175-191.

[7] D. Wienke, C. Lucasius, G. Kateman, Multicriteria target vector optimization of analysitical procdures using genetic algorithms, Anal. Chim. Acta 265 (1992) 211-225.

[8] E. Sivertsen, F. Bjerke, T. Almøy, V. Segtnan, T. Næs, Multivariate optimization by visual inspection, Chemom. Intell. Lab. Syst. 85 (2007) 110-118.

[9] J.H. Kalivas, G.G. Siano, E. Andries, H.C. Goicoechea, Calibration maintenance and transfer using Tikhonov regularization approaches, Appl. Spectrosc. 63 (2009) 800-809.

[10] M.R. Kunz, J.H. Kalivas, E. Andries, Model updating for spectral calibration maintenance and transfer using 1-norm variants of Tikhonov regularization, Anal. Chem. 82 (2010) 36423649 .

[11] J.A. Farrell, K. Higgins, J.H. Kalivas, Updating a near-infrared multivariate calibration model former with lab-prepared pharmaceutical tablet types to new tablet types in full production, J. Pharm. Biomed. Anal. 61 (2012) 114-121. 
[12] P. Teppola, V.-M. Taavitsainen, Parsimonious and robust multivariate calibration with rational function Least Absolute Shrinkage and Selection Operator and rational function Elastic Net, Anal. Chim. Acta 768 (2013) 57-68.

[13] S.R. Delwiche, J.B. Reeves, III, A graphical method to evaluate spectral preprocessing in multivariate regression calibrtions: Example with Savitzky-Golay filters ad partial least squares regression, Appl. Spectrosc. 64 (2010) 73-82

[14] B.V. Dasarathy, A representative bibliography of surveys in the information fusion domain, Inf. Fusion 11 (2010) 299-300.

[15] L.A. Klein, Sensor and Data Fusion Concepts and Applications, SPIE Optical Engineering Press, Bellingham, 1999.

[16] E. Borràs, J. Ferré, R. Boqué, M. Mestres, L, Aceña, O. Busto, Data fusion methodologies for food and beverage authentication and quality assessment - A review, Anal. Chim. Acta 891 (2015) 1-14.

[17] K. Hérberger, Sum of ranking differences compares methods or models fairly, Trends Anal. Chem. 29 (2010) 101-109.

[18] K. Hérberger, K. Kollar-Hunek, Sum of ranking differences for method discrimination and its validation: comparison of ranks with random numbers, J. Chemom. 25 (2011) 151-158.

[19] J.H. Kalivas, K. Hérberger, E. Andries, Sum of ranking differences (SRD) to ensemble multivariate calibration model merits for tuning parameter selection and comparing calibration methods, Anal. Chim. Acta 869 (2015) 21-33.

[20] C.M.R. Ginn, P. Willett, J. Bradshaw, Combination of molecular similarity measures using data fusion, Perspectives in Drug Discovery and Design 20 (2000) 1-16.

[21] P. Willett, Combination of similarity rankings using data fusion, J. Chem. Inf. Model. 53 (2013) 1-10.

[22] W. Ni, S.D. Brown, R. Man, Stacked partial least squares regression analysis for spectral calibration and prediction, J. Chemom. 23 (2009) 505-517. 
[23] W. Ni, S.D. Brown, R. Man, Data fusion in multivariate calibration transfer, Anal. Chim. Acta 661 (2010)133-142.

[24] K. Helland, H.E. Berntsen, O.S. Borgen, H. Martens, Recursive algorithm for partial least squares regression, Chemom. Intell. Lab. Sys. 14 (1991) 129-137.

[25] P. Shahbazikhah, J.H. Kalivas, A consensus modeling approach to update a spectroscopic calibration, Chemom. Intell. Lab. Sys. 120 (2013) 142-153.

[26] J.H. Kalivas, J. Palmer, Characterizing multivariate calibration tradeoffs (bias, variance, selectivity, and sensitivity) to select model tuning parameters, J. Chemom. 28 (2014) 347-357.

[27] A.A. Gowen, G. Downey, C. Esquerre, C.P. O’Donnell, Preventing over-fitting in PLS calibration models of near-infrared (NIR) spectroscopy data using regression coefficients, J. Chemom. 25 (2011) 375-381.

[28] S. Takahama, A.M. Dillner, Model selection for partial least squares calibration and implictions for analysis of atmospheric organic aerosol samples with mid-infrared spectroscopy, J. Chemom. 29 (2015) 659-668.

[29] K. Faber, B.R. Kowalski, Propagation of measurement errors for the validation of prediction obtained by principal component regression and partial least squares, J. Chemom. 11 (1997) 181238.

[30] J.B. Forrester, J.H. Kalivas, Ridge regression optimization using a harmonious approach, J. Chemom. 18 (2004) 372-384.

[31] F. Allegrini, P.D. Wentzel, A.C. Olivieri, Generalized error-dependent prediction uncertainty in multivariate calibration, Anal. Chim. Acta 903 (2016) 51-60.

[32] R.A. van den Berg, H.C.J. Hoefsloot, J.A. Westerhuis, A.K. Smilde, M.J. van der Werf, Centering scaling, and transformations: improving the biological information content of metabolomics data, BMC Genomics 7 (2006) 1-15, http://www.biomedcentral.com/14712164/7/142. 
[33] K. Kollár-Hunek, K. Héberger, Method of model comparison by sum of ranking differences in cases of repeated observations (ties), Chemom. Intell. Lab. Syst. 127 (2013) 139-146.

[34] J.A. Koziol, Sums of ranking differences and inversion numbers for method discrimination, J. Chemom. 27 (2013) 165-169.[35] Download address:

http://www.isu.edu/chem/people/faculty/kalijohn/ (assessed November 2015).

[36] M. Dyrby, S.B. Engelsen, L. Nørgaard, M. Bruhn, L. Lundesberg-Nielsen, Chemometric quantitation of the active substance (containing $\mathrm{C} \equiv \mathrm{N}$ ) in a pharmaceutical tablet using nearinfrared (NIR) transmittance and NIR FT-Raman spectra, Appl. Spectrosc. 56 (2002) 579-585.

[37] Download address: http://www.models.life.ku.dk/datasets accessed March 15 (accessed November 2015).

[38] V. Tafintseva, K. Tøndel, A. Ponosov, H. Martens, Global structure of sloppiness in a nonlinear model, J. Chemom. 28 (2014) 64-655. 


\section{Table 1}

Model quality measure values for TR models selected by the three fusion rules SRD, sum, and median.

\begin{tabular}{|c|c|c|c|c|c|}
\hline \multirow{2}{*}{ Process } & $\begin{array}{c}\text { Fusion } \\
\text { Rule }\end{array}$ & $\begin{array}{c}\text { Tuning Parameter } \\
\boldsymbol{\eta}, \boldsymbol{\lambda}\end{array}$ & $\mathbf{R M S E V}$ & $\mathbf{R}_{\text {Val }}^{2}$ & $\|\hat{\mathbf{b}}\|_{2}$ \\
\hline & SRD & $518(5), 28.9(21)$ & 0.258 & 0.967 & 1.67 \\
All models & Sum & $1000(1), 0.702(42)$ & 0.281 & 0.959 & 3.24 \\
& Median & $1000(1), 0.588(43)$ & 0.285 & 0.956 & 3.61 \\
\hline \multirow{2}{*}{ Thresholds } & SRD & $518(5), 14.3(25)$ & 0.264 & 0.967 & 2.09 \\
$(50$ and 10 \%) & Sum & $373(7), 1.70(37)$ & 0.269 & 0.965 & 2.49 \\
& Median & $720(3), 0.588(43)$ & 0.285 & 0.956 & 3.61 \\
\hline \multirow{2}{*}{ Iterative } & SRD & $72.0(17), 1.00(40)$ & 0.273 & 0.963 & 2.69 \\
\hline
\end{tabular}

${ }^{a}$ Values in parenthesis are respective tuning parameter indices relative to figure axis values. 


\section{Table 2}

Model quality measure values for PLS models selected by the three fusion rules SRD, sum, and median.

\begin{tabular}{|c|c|c|c|c|c|}
\hline \multirow{2}{*}{ Process } & $\begin{array}{c}\text { Fusion } \\
\text { Rule }\end{array}$ & $\begin{array}{c}\text { Tuning Parameter } \\
\boldsymbol{\eta}, \mathbf{L V s}\end{array}$ & $\mathbf{R M S E V}$ & $\mathbf{R}_{\text {Val }}^{2}$ & $\|\hat{\mathbf{b}}\|_{2}$ \\
\hline \multirow{3}{*}{ All models } & SRD & $848(2), 4$ & 0.339 & 0.938 & 4.64 \\
& Sum & $1000(1), 5$ & 0.371 & 0.920 & 10.2 \\
& Median & $1000(1), 21$ & 0.488 & 0.875 & 89.9 \\
\hline \multirow{2}{*}{ Thresholds } & SRD & $1000(1), 4$ & 0.339 & 0.938 & 4.64 \\
$(50$ and 10 \%) & Sum & $1000(1), 5$ & 0.371 & 0.920 & 10.2 \\
& Median & $1000(1), 5$ & 0.371 & 0.920 & 10.2 \\
\hline Iterative & SRD & $51.8(19), 27$ & 0.474 & 0.870 & 119 \\
\hline
\end{tabular}

${ }^{a}$ Values in parenthesis are respective tuning parameter indices relative to figure axis values. 


\section{Figure Captions}

Fig. 1. Graphical characterization of the SRD process using a model quality matrix with five quality measures evaluating four models. The SRD target is maximization.

Fig. 2. Graphical characterization of fusion rules being used to rank models over a set of model quality measures.

Fig. 3. Thresholding to reduce the number of models for the fusion rules. Shown is the $1-\mathrm{R}^{2}{ }_{M}$ landscape of values using the TR model updating method in equation (1). The best $50 \%$ are retained followed by removal of the top $10 \%$ models. The models remaining are then passed on to the fusion rules.

Fig. 4. Log scale images of model quality measure landscapes for the TR models (color bar values correspond to actual calculated values). (a) RMSEC for primary calibration samples, (b) RMSEM for the secondary update samples, (c) RMSEV for secondary validation samples, and (d) model vector $\mathrm{L}_{2}$ norm. In all landscapes, the tuning parameter values decrease from left to right and bottom to top.

Fig. 5. Log scale images of the RMSEV landscapes (color bar values correspond to actual calculated values) with five lowest ranked models using fusion rules SRD (magenta asterisk), sum (white plus sign), and median (red circles) for (a) all TR models, (b) TR models passing 50 and $10 \%$ thresholds on $\mathrm{R}^{2}$ values for the updating set, and (c) all PLS models. In all landscapes, the tuning parameter values decrease from left to right and bottom to top. 
Fig. 6. Log scale TR RMSEV images (color bar values correspond to actual calculated values) for (a) 332 models (grey squares) identified by SRD with the rank threshold noted in text (lowest ranked five models distinguished by magenta asterisks) and (b) four models passing the rank threshold after using SRD on the models in (a). In both landscapes, the tuning parameter values decrease from left to right and bottom to top.

Fig. 7. Log scale images of model quality measure landscapes for the PLS models (color bar values correspond to actual calculated values). (a) RMSEC for primary calibration samples, (b) RMSEM for the secondary update samples, (c) RMSEV for secondary validation samples, and (d) model vector $L_{2}$ norm. In all landscapes, the tuning parameter values decrease from left to right and bottom to top. 


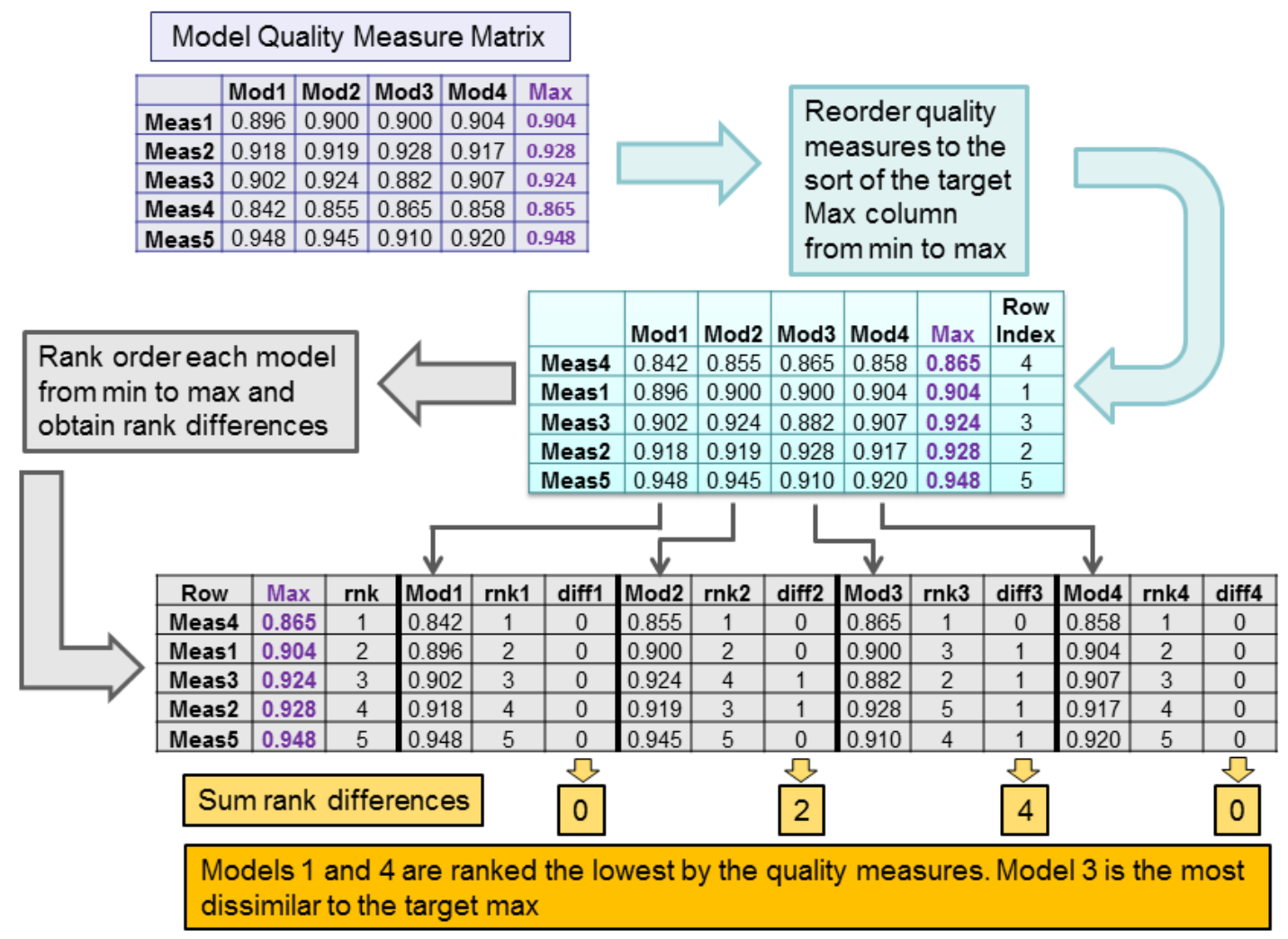

Figure 1 


\begin{tabular}{|c|c|c|c|c|}
\hline & Model 1 & Model 2 & $\ldots$ & Model $m$ \\
\hline \multicolumn{5}{|l|}{ Measure 1} \\
\hline \multicolumn{5}{|l|}{ Measure 2} \\
\hline \multicolumn{5}{|l|}{$\vdots$} \\
\hline \multicolumn{5}{|l|}{ Measure $n$} \\
\hline & b & 5 & $\sqrt{3}$ & $\sqrt{3}$ \\
\hline Fusion rule & $\begin{array}{l}\text { fused } \\
\text { score 1 }\end{array}$ & $\begin{array}{l}\text { fused } \\
\text { score 2 }\end{array}$ & $\cdots$ & $\begin{array}{l}\text { fused } \\
\text { score } m\end{array}$ \\
\hline
\end{tabular}

Fused scores are ranked and lowest rank are best models

Figure 2 




Figure 3 

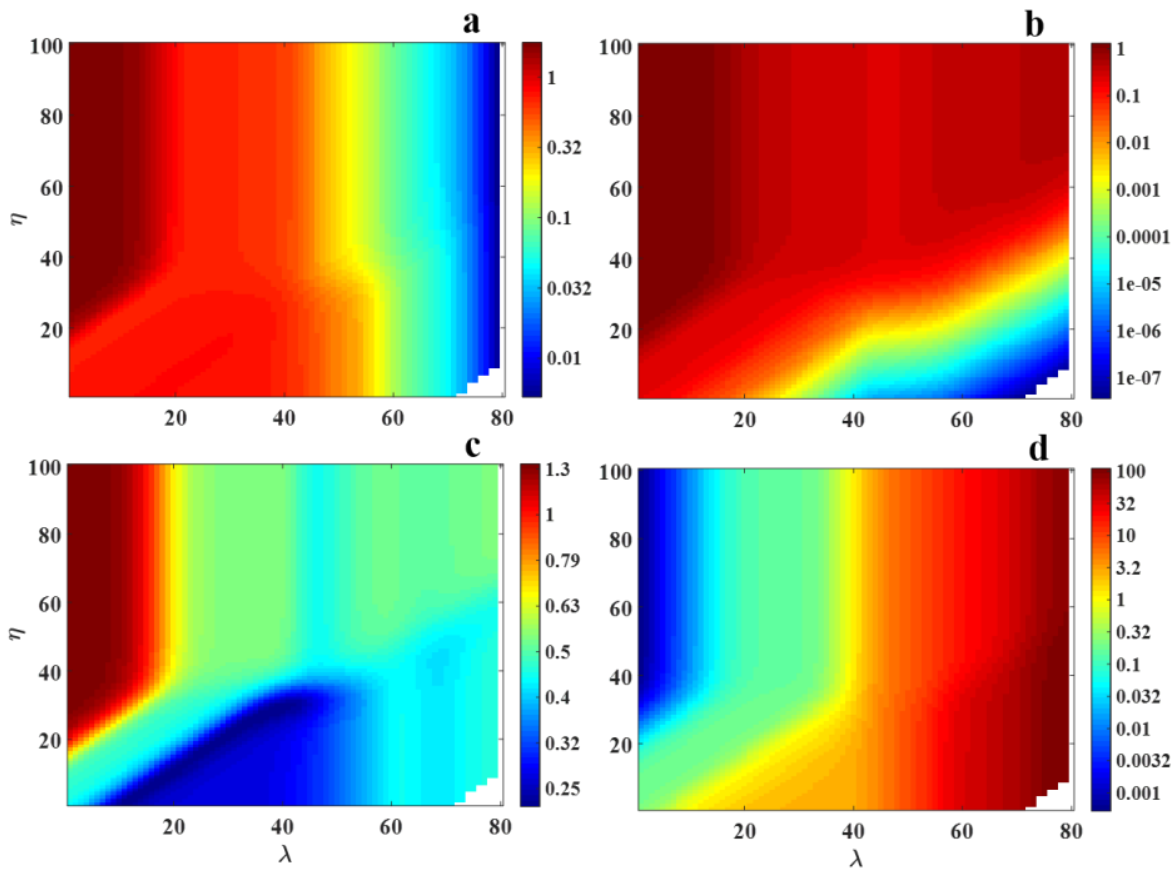

Figure 4 

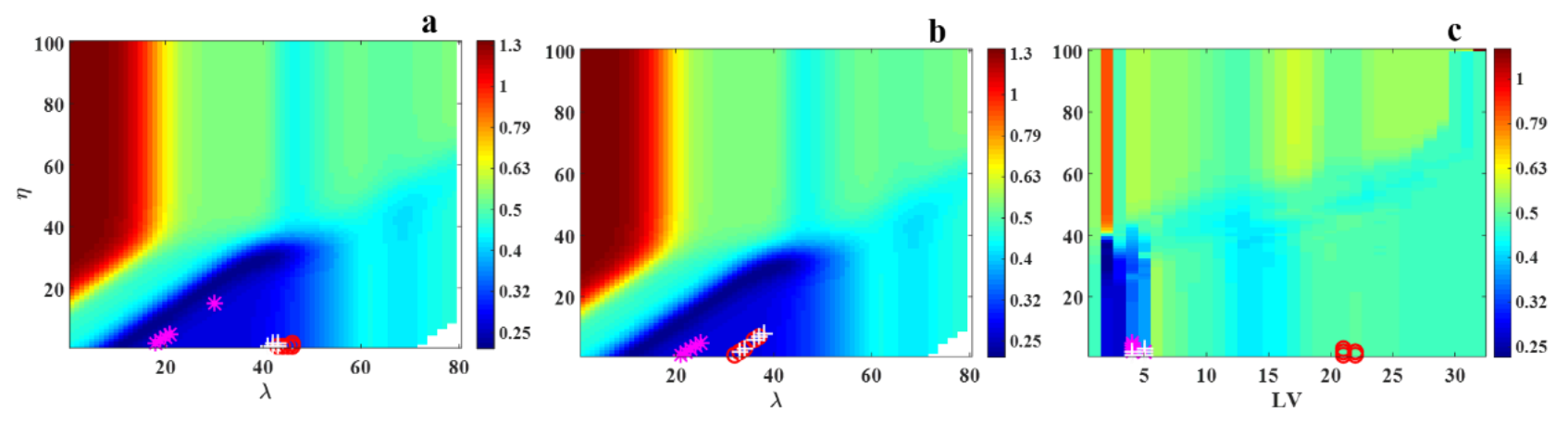

Figure 5 

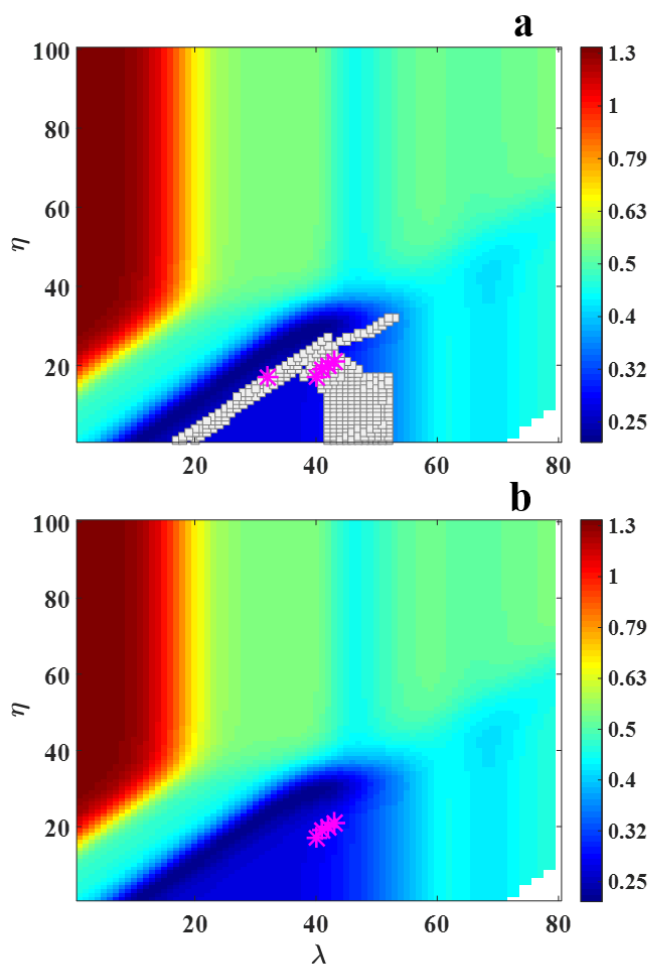

Figure 6 

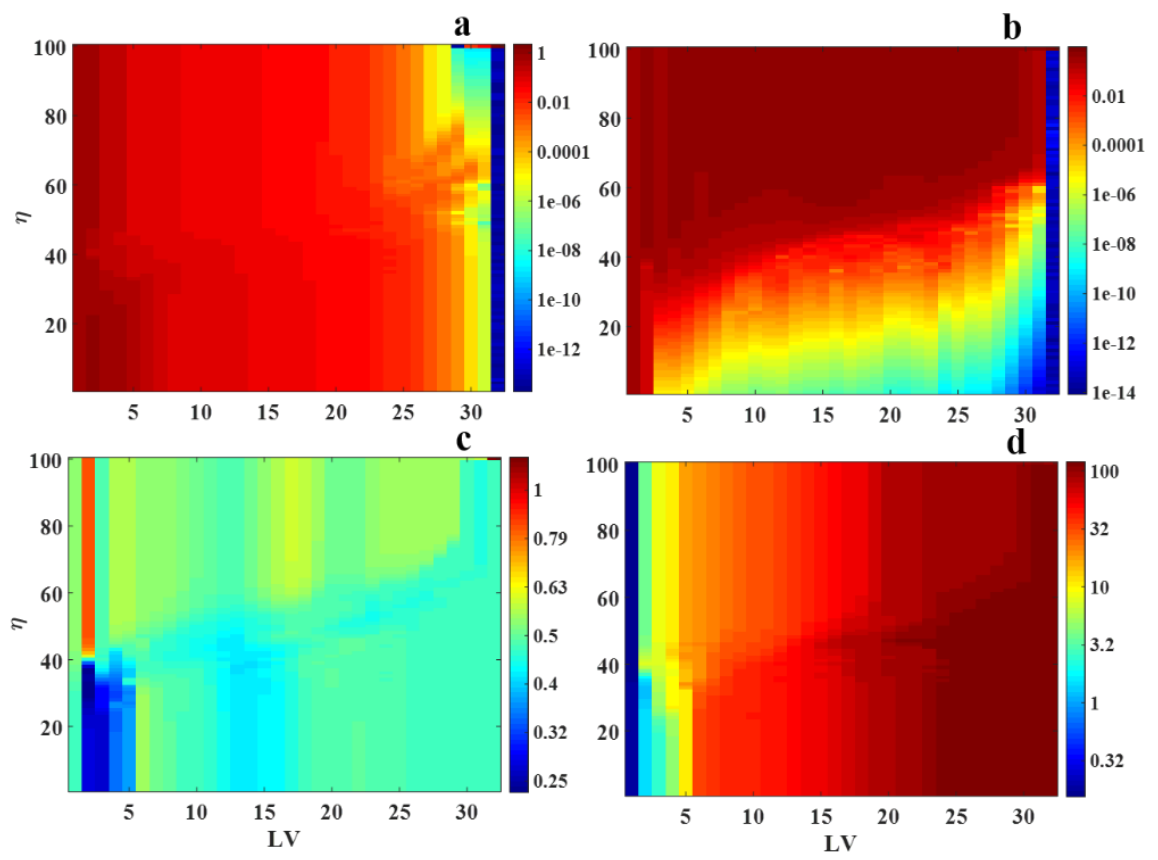

Figure 7 


\begin{tabular}{|c|c|c|c|c|}
\hline & Model1 & Model 2 & $\ldots$ & Model $m$ \\
\hline \multicolumn{5}{|l|}{ Measure 1} \\
\hline \multicolumn{5}{|l|}{ Measure 2} \\
\hline \multicolumn{5}{|l|}{ : } \\
\hline \multicolumn{5}{|l|}{ Measure $n$} \\
\hline & $\sqrt{2}$ & 5 & $\sqrt{3}$ & 万 \\
\hline Fusion rule & $\begin{array}{l}\text { fused } \\
\text { score } 1\end{array}$ & $\begin{array}{l}\text { fused } \\
\text { score } 2\end{array}$ & ... & $\begin{array}{l}\text { fused } \\
\text { score } m\end{array}$ \\
\hline
\end{tabular}

Fused scores are ranked and lowest rank are best models

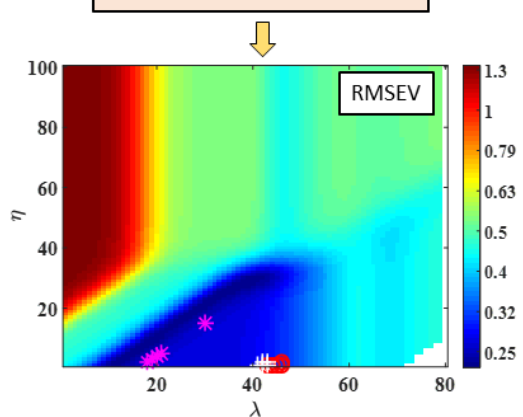

